FOUNDATIONS OF COMPUTING AND DECISION SCIENCES

Vol. 37

(2012)

No. $]$

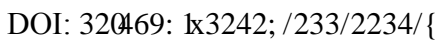

\title{
A SURVEY OF GRAPH COLORING - ITS TYPES, METHODS AND APPLICATIONS
}

\author{
Piotr FORMANOWICZ ${ }^{1,2}$, Krzysztof TANA $\dot{S}^{1}$
}

\begin{abstract}
Graph coloring is one of the best known, popular and extensively researched subject in the field of graph theory, having many applications and conjectures, which are still open and studied by various mathematicians and computer scientists along the world. In this paper we present a survey of graph coloring as an important subfield of graph theory, describing various methods of the coloring, and a list of problems and conjectures associated with them. Lastly, we turn our attention to cubic graphs, a class of graphs, which has been found to be very interesting to study and color. A brief review of graph coloring methods (in Polish) was given by Kubale in [32] and a more detailed one in a book by the same author. We extend this review and explore the field of graph coloring further, describing various results obtained by other authors and show some interesting applications of this field of graph theory.
\end{abstract}

Keywords: graph coloring, vertex coloring, edge coloring, complexity, algorithms

\section{Introduction}

Graph coloring is one of the most important, well-known and studied subfields of graph theory. An evidence of this can be found in various papers and books, in which the coloring is studied, and the problems and conjectures associated with this field of research are being described and solved. Good examples of such works are [27] and [28]. In the following sections of this paper, we describe a brief history of graph coloring and give a tour through types of coloring, problems and conjectures associated with them, and applications. We gather various results in this field of study, providing the reader with an outline of graph coloring, its types, properties and

\footnotetext{
${ }^{1}$ Poznań University of Technology, Institute of Computing Science

${ }^{2}$ Institute of Bioorganic Chemistry, Polish Academy of Sciences

${ }^{\dagger}$ Corresponding author; e-mail: Krzysztof.Tanas@cs.put.poznan.pl
} 
papers associated with previous research in this field. Then, we turn our attention to algorithmic approach to solving several presented types of graph coloring and problems connected with them.

\section{The Four Color Problem - an inspiration for development of graph coloring}

The field of graph coloring, and mathematical problems associated with this field of study, fascinated mathematicians for a long time. In the middle of the nineteenth century, it was found that an administrative map of England, with all counties, can be painted using only 4 colors. Every two adjacent (sharing a border) counties were assigned different colors in this drawing. It became an interesting problem for many scientists whether it is possible to color any possible political map satisfying the above conditions, using only four colors. What is more, in 1880 Tait proved in [41] that the Four Color Theorem is equivalent to the conjecture saying that every cubic map (every cubic planar graph without cutting edges) has a proper edge coloring with 3 colors. The problem was not solved until computer era. It was solved in 1976 by Appel and Haken in [5]. The above authors have divided the problem into many subclasses of maps, and written a computer algorithm to check if the map can be four-colored in all possible cases.

\section{What can be colored in a graph?}

In this section we will take the reader through a tour of the graph coloring, showing various types of objects, which can be colored in graphs, starting from the most simple and common ones, and then going through more sophisticated methods of graph coloring.

\subsection{Vertex coloring}

A proper vertex coloring problem for a given graph $G$ is to color all the vertices of the graph with different colors in such a way that any two adjacent (having an edge connecting them) vertices of $G$ have assigned different colors. In terms of graph theory, a proper vertex coloring with $k$ colors is a mapping $f: V(G) \rightarrow \mathbb{N}$ such that $: \forall_{v_{i}, v_{j} \in V(G), i \neq j} \exists\left(e_{i}, e_{j}\right) \Rightarrow f(i) \neq f(j)$.

A sample of a proper vertex coloring is shown in Figure 1.

Vertex coloring of graphs can represent a mathematical model of various resource assignments. An example of such a problem is to assign frequencies for stations of radio, or mobile phone network. Stations, which are in broadcasting range (and so their signals would interfere with each other) must be assigned different frequencies. 


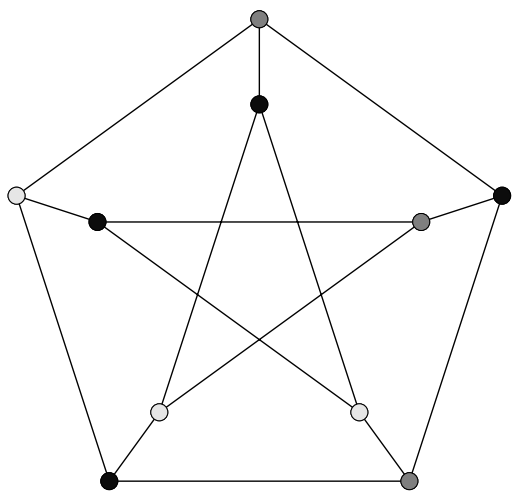

Figure 1: A proper vertex coloring.

To solve this problem, a mathematical model of the connection network is constructed, where vertices represent stations, and edges between them show conflicts (that is, pairs of stations, which need to be given different frequencies). The model itself is a graph with vertex coloring. This application of vertex coloring has been extenstively studied in many papers, among others in [11] and [44].

The minimum number of colors, for which exists a proper vertex coloring for a given graph $G$ is known as the chromatic number of the graph $G$ and is denoted by $\chi(G)$. From algorithmic point of view and for possible applications the following fact is especially important.

Fact 3.1. The vertex coloring problem is NP-complete [35].

\subsubsection{Some selected interesting types of vertex coloring}

In this chapter we take a brief tour of various interesting types of vertex coloring other than the standard (proper) coloring, and describe their examples and properties.

Equitable vertex coloring. An equitable vertex k-coloring of a graph $G=$ $(V, E)$ is a proper vertex coloring, which assigns colors from $\{1, \ldots, k\}$ to vertices in such a way that for every pair of colors $k_{a}, k_{b}, a \neq b$ the numbers of vertices painted with color $a$ and color $b$ differ by at most one. The smallest number, for which there exists an equitable vertex coloring of the graph $G$ is known as the equitable chromatic number and is denoted by $\chi_{=}(G)$.

It was proven by Hajnal and Szemeredi, that a graph $G$ has a $k$-equitable vertex coloring if $k \geq \Delta(G)+1$, where $\Delta$ is the maximum degree of the graph [21].

However, Hajnal and Szemeredi's results did not produce an effective algorithm which can find equitable vertex coloring of a given graph. A quick such an algorithm has been discovered recently by Kierstead, Kostochka, Mydlarz and Szemeredi [29]. 
The algorithm presented in their paper has $O\left(\Delta n^{2}\right)$ complexity, where $n$ is the number of graph's vertices.

This area of study has also been explored by Furmańczyk and Kubale [18]. They have given formulas for the equitable chromatic number of several graph classes and some graph products, and presented polynomial-time equitable coloring algorithms with suboptimal number of colors.

Fact 3.2. The equitable vertex coloring can be used as a representation of performed tasks, where every two connected vertices correspond to a pair of tasks which cannot be performed simultaneously (at the same time unit). An optimal coloring would assign task times so that the entire work could be finished in as short time as possible, with no colliding tasks. This application of equitable coloring was shown in [34].

Circular vertex coloring. Another type of a graph vertex coloring is the circular vertex coloring. In this type of coloring we are allowed to assign fractional numbers, not only integers, to vertices, and values of adjacent vertices need to fall within a certain range (having specified minimum difference, and the maximum value assigned to a vertex).

In terms of graph theory, a circular vertex k-coloring of a graph $G=(V, E)$ is a mapping $A: V \rightarrow[0, k)$ for which $1 \leq\left|A\left(V_{x}\right)-A\left(V_{y}\right)\right| \leq k-1$, for every pair of vertices $V_{x}, V_{y} \in V(G), x \neq y$.

Circular vertex coloring of graphs was originally defined by Vince in [42]. An example of a circular vertex coloring is shown in Figure 2. The minimum number for which there exists a circular vertex coloring of the graph $G$ is known as the circular chromatic number and denoted by $\chi_{C}(G)$.

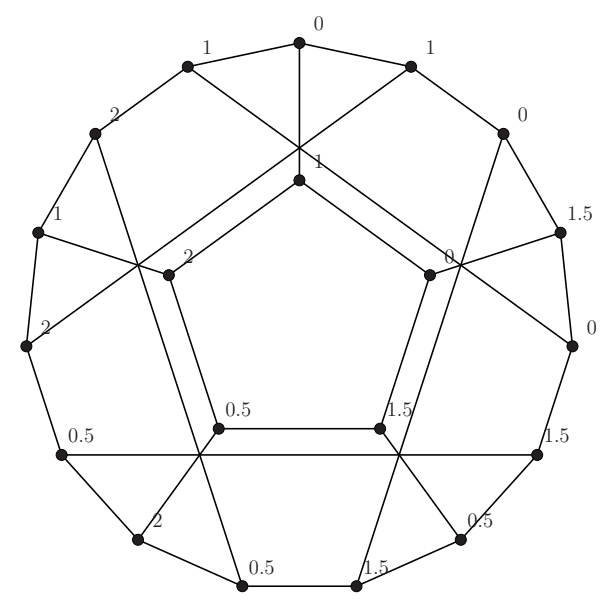

Figure 2: A circular vertex 2.5 - coloring of the Flower Snark $J_{5}$.

A brief survey of circular chromatic numbers for certain subclasses of graphs was 
given in [31]. An interesting survey, describing many properties and open problems associated with the circular chromatic index was presented by Zhu [45].

The circular vertex coloring can be applied to an open shop scheduling problem, where independent jobs are processed in a shop with dedicated renewable resources [36].

Acyclic vertex coloring. An acyclic vertex coloring of a graph $G$ is a proper vertex coloring without a 2-colored (alternating) cycle. The minimum number for which a given graph $G$ contains an acyclic vertex coloring is defined as the acyclic chromatic number, $A(G)$. An example of an acyclic 3-vertex coloring is shown in Figure 3 .

It is known that $A(G) \leq 4$ when $\Delta(G)=3 \quad[20]$, and $A(G) \leq 5$ when $\Delta(G)=4$ [9]. Checking whether $A(G) \leq 3$ is a NP-complete problem [30]. Alon, McDiarmid and Reed have proven that $A(G)=O\left(\Delta(G)^{\frac{4}{3}}\right), \Delta \rightarrow \infty$ [2].

Two efficient acyclic graph coloring algorithms for graphs with maximum vertex degree 3 (one colors vertices, and the other edges) were presented by Skulrattanakulchai [40].

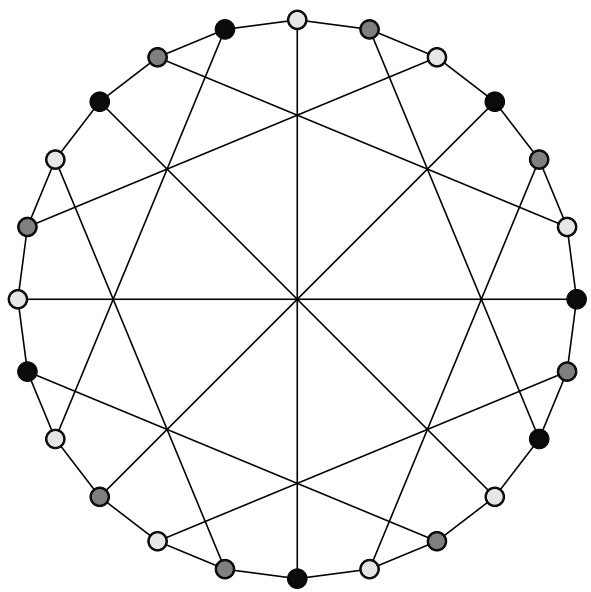

Figure 3: An acyclic 3-vertex coloring.

Star vertex coloring. A star coloring of a graph $G$ is a proper vertex coloring satisfying the condition that every path of length 4 contains vertices of at least 3 different colors. The minimum number, for which a given graph $G$ has a star coloring, or a star chromatic number is denoted as $\chi_{S}(G)$. Star coloring has been introduced by Grunbaum in [20]. A sample star 4-coloring of a 32-vertex Dyck graph is shown in Figure 4. 
Determining whether $\chi_{S}(G) \leq 3$ is NP-complete, even if we know that the graph $G$ is both planar and bipartite [1].

A survey of the star coloring numbers for several various classes of graphs was given by Fertin, Raspaud and Reed [15].

Moreover, a quick, linear-time algorithm finding optimal acyclic and star colorings for cographs has been found recently by Lyons [33]. The author has also proven that every acyclic coloring of a cograph is also a star coloring.

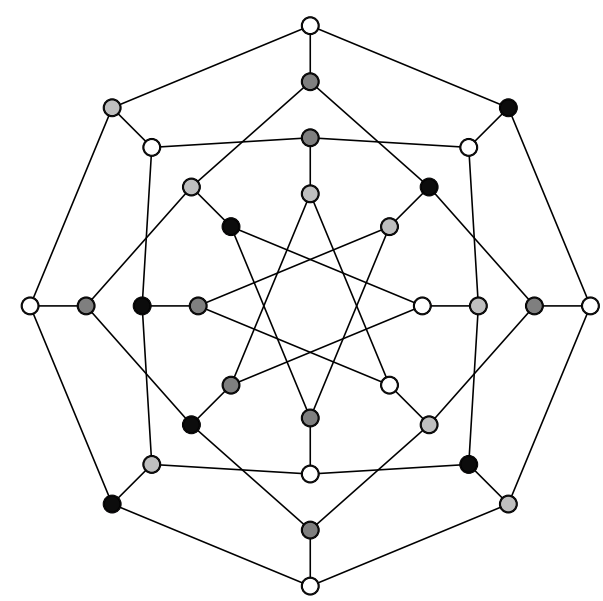

Figure 4: A star coloring.

\section{$3.2 \quad$ Edge coloring}

The other well-known and intensely studied type of graph coloring besides vertex coloring is the edge coloring. Analogically to the definition of the vertex coloring, the edge coloring of a graph $G=(V, E)$ is a mapping, which assigns a color to every edge, satisfying condition that no two edges sharing a common vertex have the same color. Mathematically, a proper (classic) edge coloring of a graph $G$ is a mapping $f: E(G) \rightarrow \mathbb{N}$ such that $\forall\left(e_{i}, e_{j} \in E(G)\right), i \neq j e_{i}, e_{j}$ are adjacent $\Rightarrow f(i) \neq f(j)$.

Two examples of edge coloring are shown in Figure 5.

Fact 3.3. It was proven by Vizing in [43] that the minimum number of colors needed to construct a proper edge coloring (the chromatic index $\chi^{\prime}(G)$ of any graph $G$ is limited to a set of two values $\{\Delta G, \Delta G+1\}$, where $\Delta(G)$ is the maximum degree of a vertex in $G$. In fact, those graphs are known as class 1, and class 2 graphs, respectively.

In the example given in Figure 5 we show edge colorings of two graphs with $\Delta(G)=3$, the left one belonging to the class 1 , with a proper 3-edge coloring, and 

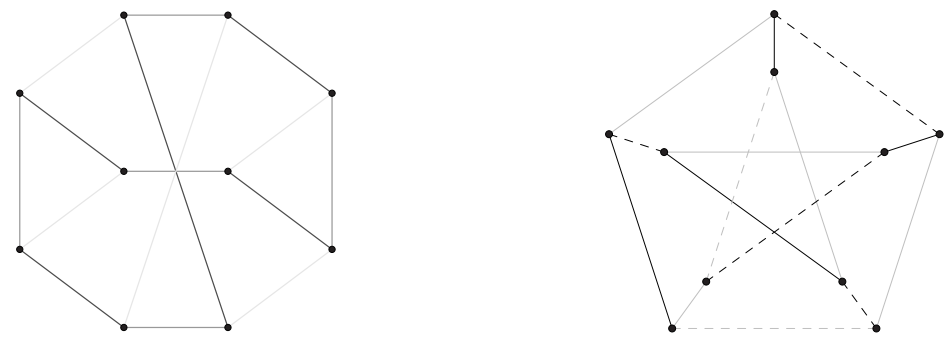

Figure 5: Two examples of an edge coloring

the other one being a class 2 graph, and thus requiring 4 colors to properly color its edges.

Edge coloring of graphs has various applications and is linked with many interesting research problems, such as the route planning problem, transfer assignment in computer networks, and many others. A nice review of edge-coloring problems and its applications was given by Nakano, Zhou and Nishizeki [38].

The minimum number of colors, for which there exists a proper edge coloring for a given graph $G$ is known as the chromatic index of the graph $G$ and is denoted by $\chi^{\prime}(G)$. The following fact describes an important property of edge coloring.

Fact 3.4. It is known that determining the chromatic index of a given graph is NPcomplete. It is true even if we limit only to cubic (3-regular) graphs. [23]

\subsubsection{Special types of edge coloring}

As we did earlier in the paper, we now turn our attention to a few chosen interesting non-standard types of edge coloring.

Circular edge coloring. Analogically to the circular vertex coloring, the edges of a graph could be colored in such a way that no two adjacent (sharing a common vertex) edges have assigned values differing by no less than some specified number. A mapping $C: E(G) \rightarrow[0, k)$ such that for every two adjacent edges $e_{a}, e_{b} \in E(G)$ $1 \leq|C(a)-C(b)| \leq k-1$ is called the circular k-edge coloring of the graph $G$. The minimum number, for which there exists a circular edge coloring of a given graph $G$ is known as the circular chromatic index of the graph $G$ and is denoted by $\chi_{C}^{\prime}(G)$.

Figure 6 shows an example of a circular edge coloring. This is a $\frac{11}{3}$-edge coloring of the Petersen Graph.

Fact 3.5. A circular $k$-edge coloring of any graph $G$ is a circular $k$-vertex coloring of the line graph $L(G)$. Thus $\chi_{C}^{\prime}(G)=\chi_{C}(L(G))$.

An approximate algorithm for circular edge coloring of graphs was given by Janczewski, Kuszner, Małafiejski and Nadolski [26]. 


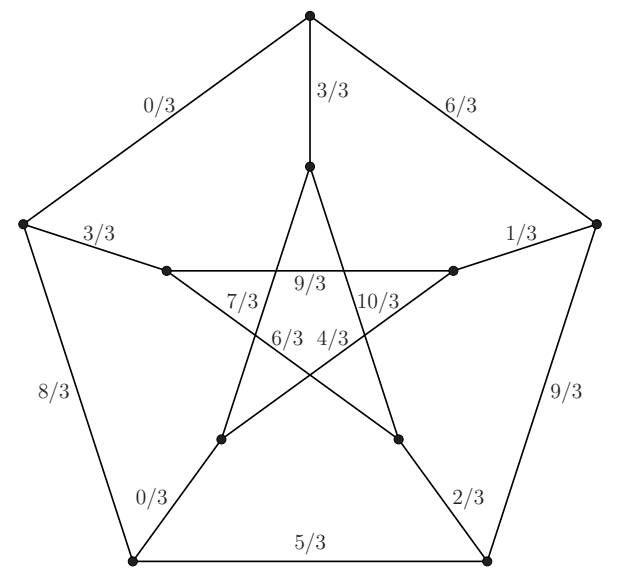

Figure 6: A circular (11/3)-edge coloring of the Petersen graph.

Acyclic edge coloring. In a similar way as with the vertex coloring, the edges of a graph could also be colored in such a way that there does not exist a 2-colored (alternating) cycle. The minimum number of colors needed to color the edges of a given graph $G$ acyclically is called the acyclic chromatic index of the graph $G$, and is denoted by $A^{\prime}(G)$.

It is known that $A^{\prime} G \leq 16 \Delta(G)$ for any graph $G$, and there exists a constant $c$ such that $A^{\prime}(G) \leq \Delta(G)+2$ for every graph $G$ of girth (length of the graph's shortest cycle) greater or equal to $c \Delta(G) \log \Delta(G)$. Moreover, $A^{\prime}(G) \leq \Delta(G)+2$ for almost every regular graph [3].

Alon and Zaks have proven that determining the acyclic chromatic index of an arbitrary graph $G$ is NP-complete. However, if the girth of graph $G$ is large enough in relation to $\Delta(G)$, there does exist a polynomial-time algorithm creating an acyclic coloring of $G$ with at most $\Delta(G)+2$ colors [4].

For planar graphs, in was conjectured that for sufficiently large $\Delta(G), A^{\prime}(G)=$ $\Delta(G)$. What is more, $A^{\prime}(G) \leq \Delta(G)+25[10]$.

Berge-Fulkerson and Fan-Raspaud coloring. Another two interesting types of edge coloring are the Berge-Fulkerson and Fan-Raspaud colorings. In both cases we are working on bridgeless cubic graphs, that is, graphs with all vertices of degree 3 , and without bridges (cutting edges). This class of graphs could represent a mathematical model of a cubic computer network, where vertices represent computers, and the edges correspond to network connections (cables) connecting them. The cubic network is an effective network topology, due to the constant vertex degree, which allows relatively easy implementation of algorithms operating in the whole network. The graph representing the network would almost certainly be bridgeless, as it is generally unwise to connect computers in such a way that a failure of just 
one connection would disconnect the network into separate components, and make communication between some computers and the others unavailable.

Now let us suppose that a computer network constructed in the above way, represented mathematically as a bridgeless cubic graph is performing a research project, which consists of $m$ parallel computations, which must be performed by pairs of computers, where each pair shares a network connection. For every of the $m$ computations, each computer in the network must be assigned to exactly one connected pair working on this computation, but the connections (or cables) have a transfer limit, so that any cable can simultaneously transfer data for at most 2 computations. Assigning one connection to three or more computations would be technically unavailable, or would reduce the speed of the whole project below satisfactory level, or would damage the network connection (due to overheating, for example).

In graph theory terms, we need to construct an edge coloring, consisting of $m$ perfect matchings, where every edge belongs to at most two of them. For $m=6$, we would have a Berge-Fulkerson coloring, and for $m=3$ - a Fan-Raspaud coloring. The origins of such types of graph coloring were given in [17] and [14], respectively. They formulated conjectures, which state that every bridgeless cubic graph can be colored in such a way. No proof, or counterexample has been found yet.

A sample Berge-Fulkerson and Fan-Raspaud colorings of the Petersen graph are shown in Figure 7 . Every perfect matching, representing one part of the research project computed by the network, is marked with a distinct color of the edges.
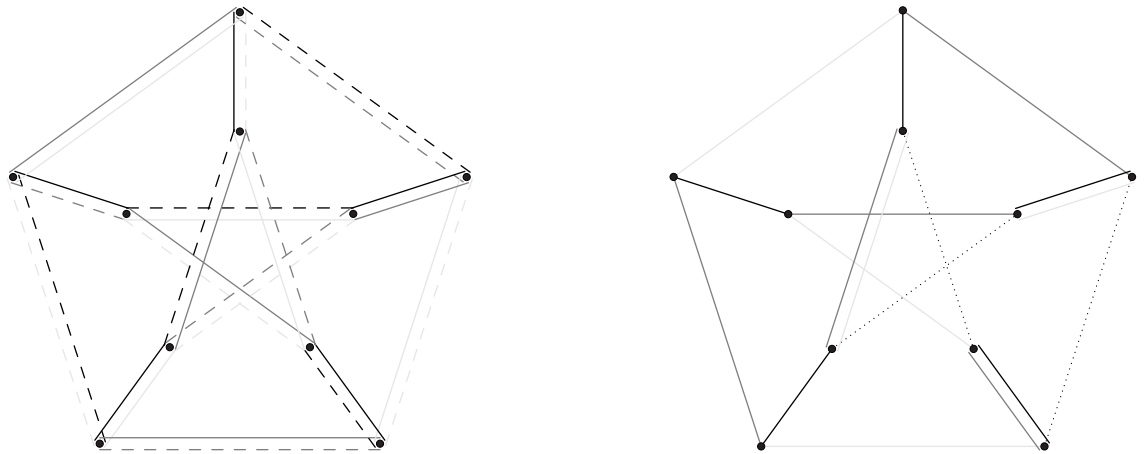

Figure 7: A Berge-Fulkerson coloring (left) and a Fan-Raspaud coloring (right) of the Petersen graph. The dotted lines represent empty edges.

Fact 3.6. It is obvious that every proper 3-edge coloring is a Fan-Raspaud coloring, and duplicating any such coloring (adding a second triple of colors to edges in the same way as in the original coloring) would produce a Berge-Fulkerson coloring. However, some bridgeless cubic graphs do not have a proper 3-edge coloring. These graphs are called snarks. A classic example of a snark is the Petersen graph. 
Fact 3.7. Holyer has proven that determinig whether a bridgeless cubic graph has a proper 3-edge coloring is an NP-complete problem. [23].

Due to the NP-completeness of a classic 3-edge coloring problem, it is suspected that finding a Fan-Raspaud coloring is also NP-complete, what would imply that there does not exist a deterministic polynomial time algorithm finding such colorings (unless $\mathrm{P}=\mathrm{NP}$ ). So it is a good idea to use randomized algorithms instead. A randomized algorithm constructing Fan-Raspaud colorings for a given cubic graph is presented in [16].

\subsection{Face and map coloring}

Aside from two most common types of graph coloring, the vertex coloring and edge coloring, there exist various other objects in graphs that can be colored. Next method shown in this paper will be the face coloring, also known as map coloring.

The face coloring is the method used to color areas on a political map, and so it is the coloring associated with the Four Color theorem. This type of coloring requires a graph to be planar, what means the graph can be drawn on a 2-dimensional plane without intersections between edges.

In fact, a map coloring can be done by creating the dual graph, in which every vertex represents one face of the map (including the outermost face), and any two vertices are connected if and only if faces represented by them are adjacent. A sample graph and its dual graph is drawn in Figure 8.

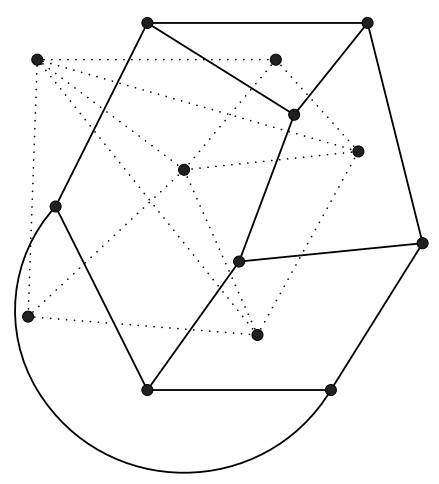

Figure 8: A graph (solid lines) and its dual graph (dotted lines).

Fact 3.8. The face coloring of planar graphs is a NP-complete problem [19]. 


\subsection{List coloring}

Another interesting type of graph coloring is the list coloring. In list coloring, unlike the classic vertex or edge coloring, the possible color assigned to each object (vertex or edge) of a given graph is limited to a restricted set, called a list. Every vertex or edge could have different list. In terms of graph theory, a vertex list coloring is a mapping, which assigns every vertex a color from the vertex's list such that no two adjacent vertices have the same color. The edge list coloring can be defined analogically, in this case no two incident edges are painted with the same color. The list coloring of graphs can represent a mathematical model of job scheduling, where vertices represent employees (or machines), which will have assignes jobs, and the lists describe possible jobs for a given vertex. The list coloring, and a detailed review of its properties and conjectures is shown in papers [12] and [6].

Fact 3.9. List coloring is NP-complete even for some classes of graphs for which classic vertex coloring is solvable in linear time, for example the interval graphs [7].

\subsection{Path coloring}

A path coloring of a given graph is a specific type of edge coloring. Here, we need to color certain paths linking some certain pairs of vertices, and where each path contains a specific set of edges, satisfying the condition that no two paths containing the same edge (or in some versions of the path coloring it is required for the same-color paths to pass through disjoint sets of vertices) of the graph or multigraph (depending on the problem to solve there may be several edges connecting the same vertices). Path coloring of graphs can be used as a mathematical model of call scheduling in a network, where a set of requests to transfer data between certain nodes is to be handled, and paths which have common edges, or intersecting vertices, must be given different colors. Another possible application of path coloring is the coloring of tourist routes. The routes intersecting at any crossing point must be given different colors. In this case, a multigraph is colored, because certain routes may have common segments, represented by edges with more than one color. An overview of path coloring and its complexity results was shown by Erlebach and Jansen [13].

An example of a path coloring is shown in Figure 9.

\subsection{Total coloring}

In the total coloring of a graph, vertices and edges are colored simultaneously. Every two adjacent vertices, any two edges having a common vertex, as well as every incident pair (vertex, edge) must be assigned different colors. A sample total coloring of a graph is shown in Figure 10.

The minimum number of colors needed to construct a total coloring for a given graph $G$ is known as the total chromatic number and is denoted by $\chi^{\prime \prime}(G)$. 


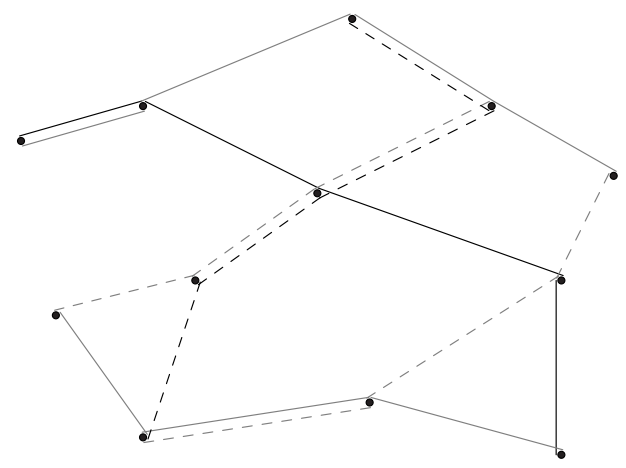

Figure 9: A sample path (multi)coloring of a graph.

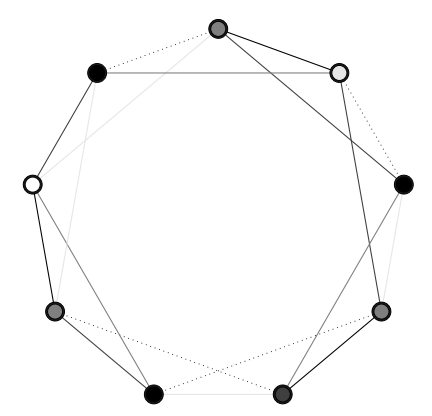

Figure 10: A total coloring.

Fact 3.10. It was conjectured by Behzad and Vizing (independently) that for every graph $G$

$\chi^{\prime \prime}(G) \leq \Delta(G)+2$.

If the above conjecture (the Total coloring conjecture) is true, then for every graph $\chi^{\prime \prime}(G) \in\{\Delta(G)+1, \Delta(G)+2\}$ and so, graphs could be divided into two classes in relation to the total chromatic number.

It was proven by probabilistical methods, that for sufficiently large graphs, there exists a bound on total chromatic index relative to the maximum vertex degree in graph, namely $\chi^{\prime \prime}(G) \leq \Delta(G)+10^{26} \quad[37]$.

A further exploration of total graph coloring, its properties, conjectures, theorems and results was presented in [24]. 


\section{An algorithmic approach to graph coloring - a view on heuris- tics}

Having already shown various types of graph coloring, and provided inforamtion about them, we now turn our attention to algorithmic methods of solving graph coloring problems. As the graph theory and coloring plays an important role in computer science and related fields of study, and algorithms working on graphs and performing various types of coloring are extensively used, constructed and further developed, we now present a brief survey of algorithmic methods of constructing various types of graph coloring. As we have already presented results on the computational complexity for several types of graph coloring, many problems turn out to be NP-complete, which means it would be difficult to construct an effective coloring algorithm for them. In fact, there is no deterministic algorithm able to find solution in polynomial time, unless it turns out that $P=N P$. Due to the above facts, we now stay aside from wellknown deterministic coloring algorithms and emphasize on heuristic ones. A glimpse of heuristics could provide information and direction, which would allow researchers to create more efficient algorithms, needing less time and/or memory, and having higher probability of success, that is, finding a solution for a given problem.

A heuristic coloring algorithm was constructed by Brelaz [8]. This algorithm constructs vertex coloring using the saturation degree - the number of different colors for which already exists a vertex adjacent to the subject vertex.

For edge coloring, a set of complexity results for many possible subclasses of graphs was presented in [38]. The heuristics for edge coloring were further explored in several other papers. A survey of edge coloring heuristics for large graphs, divided into few different methods, such as evolutionary algorithms and the cyclic, random, line and ant heuristics has been shown in [22].

For list coloring, an interesting heuristic coloring algorithm for random graphs was given in [39].

For the total coloring of graphs, some interesting results in the field of algorithmic approach appeared in [25]. The cited paper has shown algorithms for total coloring of various classes of graphs, such as the $s$-degenerate graphs and partial $k$-trees. There was also proven that $s$-degenerate graphs with sufficiently large maximum vertex degree belong to class 1 in the terms of total coloring, that is, $\chi^{\prime \prime}(G)=\Delta(G)+1$ for this class of graphs.

\section{Conclusion}

In this paper, we have presented a brief survey of the various types of graph coloring, guiding the reader through subfields of this field of study, including several types of coloring, selected important results connected to them, such as mathematical properties, conjectures, complexities and algorithms. As the field of graph coloring is one of the most important, interesting, and developing branches of graph theory, and many new properties, conjectures, proofs and algorithms are actively constructed, formu- 
lated and explored by mathematicians and computer scientists all over the world, it would be helpful to provide future researchers with information about various coloring types, its properties and applications. As the article is supposed to be read by computer scientists, we emphasize on the complexity results and algorithmic approach, especially on heuristic algorithms.

\section{References}

[1] Chappell G. Hal A. Kundgen A. Ramamurthi R. Albertson, M.O. Coloring with no 2-colored $\mathrm{p}_{4}$ 's. Electronic Journal of Combinatorics, 11(1), 2004.

[2] McDiarmid C. Reed B. Alon, N. Acyclic coloring of graphs. Random Structures and Algorithms, 2(3):277-288, 1991.

[3] Sudakov B. Zaks A. Alon, N. Acyclic edge colorings of graphs. J. Graph Theory, 37(3):157-167, 2001.

[4] Zaks A. Alon, N. Algorithmic aspects of acyclic edge colorings. Algorithmica, $32(4): 611-614,2002$.

[5] Haken W. Appel, K. Every planar graph is four-colorable. Illinois. J. Math., 21:429-490, 1976.

[6] Courtney L. Baber. An introduction to list colorings of graphs. Master's thesis, Virginia Polytechnic Institute and State University, Blacksburg, Virginia, April 2009 .

[7] Hujter M. Tuza Z. Biro, M. Precoloring extensions. i. interval graphs. Discrete Mathematics, 100:267-279, 1992.

[8] D. Brelaz. New methods to color the vertices of a graph. Comm. ACM, 22:251256, 1979.

[9] M.I. Burstein. Every 4-valent graph has an acyclic 5-coloring. Soobsc. Akad. Nauk Gruzin. SSR, 93:21-24, 1979.

[10] Havet F. Muller T. Cohen, N. Acyclic edge-colouring of planar graphs. Technical report, Institut National de Recherche en Informatique et en Automatique, March 2009.

[11] Grotschel M. Koster A. Eisenblatter, A. Frequency planning and ramifications of coloring. Technical report, Konrad-Zuse-Zentrum fur Informationstechnik Berlin, Takustrasse 7, D-14196 Berlin-Dahlem, Germany, December 2000.

[12] Rubin A.L. Taylor H. Erdos, P. Choosability in graphs. Congr. Numer., 26:125$157,1979$.

[13] Jansen K. Erlebach, T. The complexity of path coloring and call scheduling. Theor. Comp. Sci, 255:33-50, 2001. 
[14] Raspaud Andre Fan, Genghua. Fulkerson's conjecture and circuit covers. J. Comb. Theory, Ser. B, 61(1):133-138, 1994.

[15] Raspaud A. Reed B. Fertin, G. Star coloring of graphs. Journal of Graph Theory, 47(3):163-182, 2004.

[16] Tanaś K. Formanowicz, P. The Fan-Raspaud conjecture: A randomized algorithmic approach and application to the pair assignment problem in cubic networks. Int. J. Appl. Math. Comput Sci., 22(3):765-778, 2012.

[17] D.R. Fulkerson. Blocking and anti-blocking pairs of polyhedra. Math. Programming, 1:168-194, 1971.

[18] Kubale M. Furmańczyk, H. The complexity of equitable vertex colorings of graphs. Journal of Applied Computer Science, 13(2):95-106, 2005.

[19] Johnson D.S. Stockmeyer L. Garey, M.R. Some simplified np-complete graph problems. Theor. Comp. Sci., 1:237-267, 1976.

[20] B. Grunbaum. Acyclic coloring of planar graphs. Israel J. Math., 14(390-408), 1973.

[21] Szemeredi E. Hajnal, A. Proof of a conjecture of p. Erdos. In Balatonfured Proc. Colloq., editor, Combinatorial theory and its applications, II, pages 601623. North-Holland, 1970.

[22] Drechsler N. Drechsler R. Hilgemeier, M. Fast heuristics for the edge coloring of large graphs. In Euromicro Symposium on Digital System Design, 2003. Proceedings., 2003.

[23] I. Holyer. The np-completeness of edge colourings. SIAM J. Computing, 10:718$720,1981$.

[24] T.E. Huffman. Total coloring of graphs. Master's thesis, San Jose State University, 1989.

[25] S. Isobe. Algorithms for the Total Colorings of Graphs. PhD thesis, Graduate School of Information Sciences, Tohoku University, Japan, February 2002.

[26] Kuszner Ł. Małafiejski M. Nadolski A. Janczewski, R. An approximate algorithm for circular edge coloring of graphs. Zeszyty Naukowe Wydziału ETI Politechniki Gdańskiej, 2:473-479, 2003.

[27] Toft B. Jensen, T.R. Graph Coloring Problems. John Wiley and Sons, New York, 1995 .

[28] Toft B. Jensen, T.R. 25 pretty graph colouring problems. Discrete Mathematics, 229:167-169, February 2001.

[29] Kostochka A.V. Mydlarz M. Szemeredi E. Kierstead, H.A. A fast algorithm for equitable coloring. Combinatorica, 30(2):217-224, 2010. 
[30] A.V. Kostochka. Upper bounds of chromatic functions of graphs. PhD thesis, Novosibirsk, 1978.

[31] M. Kubale, editor. Contemporary Mathematics. Graph Colorings, volume 352, chapter 9, page 126. American mathematical Society, 2004.

[32] M. Kubale. Modele i metody kolorowania grafów. Część I (in Polish). Przeglad Elektrotechniczny (Electrical Review), 86:115-117, 2010.

[33] A. Lyons. Acyclic and star colorings of cographs. Discrete Appl. Math., 159(16):1842-1850, 2011.

[34] W. Meyer. Equitable coloring. American Mathematical Monthly, 80(8):920-922, 1973.

[35] Thatcher J.W. Miller, R.E., editor. Complexity of Computer Computations, chapter Reducibility among combinatorial problems, pages 85-103. Plenum Press, New York, 1972.

[36] Ghandehari M. Modarres, M. Applying circular coloring to open shop scheduling. Scientia Iranica, 15(5):652-660, October 2008.

[37] Reed B. Molloy, M. A bound on the total chromatic number. Combinatorica, 18(2), 241-280 1998.

[38] Zhou X. Nishizeki T. Nakano, S. Edge-coloring algorithms. Technical report, Graduate School of Information Sciences, Tohoku University, Sendai 980-77, Japan, 1996.

[39] Tsouros C. Satratzemi, M. A heuristic algorithm for the list coloring of a random graph. In The 7th Balkan Conference on Operational Research, Constanta, Romania, 2005.

[40] San Skulrattanakulchai. Acyclic colorings of subcubic graphs. Information processing letters, 92(4):161-167, 2004.

[41] P.G. Tait. On the coloring of maps. Proc. Royal Soc. Edinburgh, 10:501-503, 1880 .

[42] A. Vince. Star chromatic number. Journal of Graph Theory, 12(4):551-559, 1988 .

[43] V.G. Vizing. On an estimate of the chromatic class of a p-graph. Diskret. Analiz., 3:24-30, 1964.

[44] R.J. Waters. Graph Colouring and Frequency Assignment. PhD thesis, University of Nottingham, 2005.

[45] Xuding Zhu. Circular chromatic number: a survey. Discrete Math., 229:371-410, 2001.

Received Semptember, 2012 